\title{
CompuLyte
}

\section{New developments in quantitative imaging cytometry}

\author{
Elena Holden, Ed Luther \& Melvin Henriksen
}

CompuCyte Corporation (Cambridge, Massachusetts, USA) presents its iGeneration family of imaging cytometers, based on the company's proprietary laser scanning cytometry (LSC) technology. The iCyte ${ }^{\circledR}$, iCys $^{T M}$ and iColor $^{T M}$ I maging Cytometers allow precise quantification of fluorescent signal and laser light loss, as well as imaging of cellular and tissue specimens on a solid substrate.

Recent increases in the use of cell- and tissue-based experimental models in life sciences and drug discovery have fueled a demand for a combination of imaging and quantitative cytometry techniques. This demand has been met with the development of LSC, which has substantial advantages over other cellular analysis technologies, such as fluorescence image analysis (FIA) and flow cytometry $(\mathrm{FC})^{1}$.

FIA, FC and LSC can each be used to assay and quantify multiple cell components by measuring the fluorescence of cells that have been treated with one or more fluorescent dyes. FC and LSC can be used to measure light scatter in addition to fluorescence, as both result from interaction of the cells with a laser beam, as opposed to the mercury or xenon arc epi-illuminator typically used in FIA.

The analytical capabilities of FC are severely limited for many applications, particularly for analysis of adherent cells and intact tissue samples. Events requiring time resolution—such as enzyme kinetics, drug uptake or efflux — cannot be analyzed on individual cells, and subcellular fluorochrome localization cannot be quantified. Furthermore, once a cell has been measured, it cannot be reanalyzed with another probe, and the sample cannot be stored for archival preservation. In addition, applications such as assessment of the morphology of a measured cell and smallsample analysis are difficult to perform with FC.

\section{LSC product configuration}

An LSC unit contains up to three lasers of different wavelengths. Beams from these lasers are combined into a coincident path and directed through several beam-shaping optics to an inverted microscope and onto a focal plane at the specimen. A scan line is produced by a mirror in the optical path, oscillating at a nominal rate of $350 \mathrm{~Hz}$. The analysis protocol may be defined with alternate scanned wavelengths to separate incompatible excitation and emission wavelength combinations. Computer control

CompuCyte Corporation, 12 Emily Street, Cambridge, Massachusetts 02139, USA. Correspondence should be addressed to E.H. (eholden@compucyte.com).

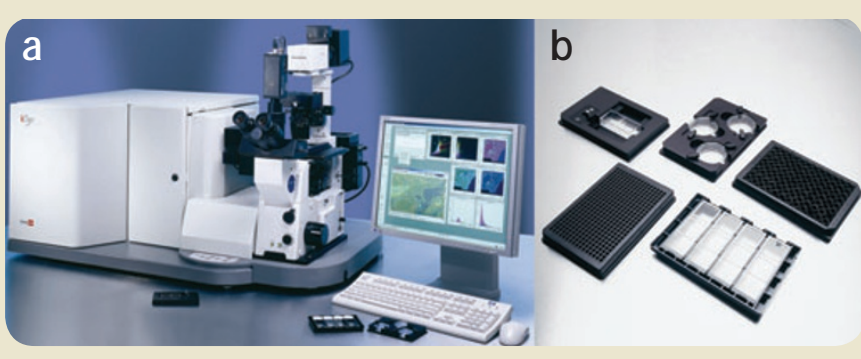

Figure $\mathbf{1}$ | The iCys research imaging cytometer. (a, b) The iCys instrument (a) and a sampling of the carrier types that can be analyzed on the system (b).

of both the specimen carrier stage and the scan mirror allows the scan coordinates to be monitored and recorded. Fluorescent energy emitted by each cell is collected by the objective lens, collimated and delivered to four photomultiplier tubes (PMTs), each detecting a specific range of wavelengths. As the laser light intersects a cell, scattered light (or absorbed light when in the light-loss mode) is collected by a lens and directed to an assembly containing one or more solid-state photosensors. The photosensor or PMT signals are converted to 14-bit pixel values (Fig. 1).

LSC allows analysis of specimens on a variety of media, including microscope slides, microtiter plates, chamber slides, petri dishes or additional user-defined carriers fitting the footprint of a microtiter plate. Autofocus is an integral part of the cytometers, minimizing operator involvement in the analysis. Although high-quality digital imaging on these systems eliminates the need for an optical microscope, microscope functions can still be provided to allow post-scan visualization of any location of interest, if an interactive research approach is needed. An optional robotic arm is also available for automatic loading of up to 45 carriers for large-scale walk-away experiments.

\section{Data analysis protocols}

Raw scan data can be saved as JPEG or 16-bit image files suitable for data processing, specimen visualization and quantification by 


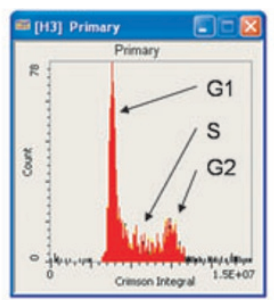

proprietary analytic software. Contours are drawn around cellular events based on the fluorescence intensity, forward scatter or excitation light transmittal. These contours may be generated automatically to select an entire cell area, or defined by the user to target specific types of events. Once the contours are generated, the software can perform a wide range of analyses on the data and produce output in the form of numerical statistics, scattergrams, histograms, expression maps and a variety of other statistical visualizations.

Analysis protocols are 'written' by graphically assembling various functional modules to establish the desired analytical workflow. The attributes of each module can be modified by highlighting the module and adjusting the desired settings. The effect of such changes on image processing can be viewed immediately in the scanned images. Customizable protocol templates are provided as frameworks for different types of assays.

\section{Performance characteristics}

The precision of LSC systems, as measured with fluorescent DNA check beads, is typically $\leq 2.5 \%$ full-width-half-max in the green, orange and far-red channels. Sensitivity is typically below 1,000 mean equivalent fluorescence (MEF). Linearity, measured as the ratio of bead singlet and doublet populations, is between 1.95 and 2.05. Relocation precision is within \pm 2 microns.

Scan field images are generated with up to 0.5 micron $\times 0.25$ micron resolution with a $40 \times$ objective lens.

\section{Cell cycle analysis applications}

The flexibility of LSC makes it suitable for several applications in basic life science research and drug discovery. Foremost among these is cell cycle analysis ${ }^{2,3}$. As in FC, the total amount of DNA per cell can be precisely and stoichiometrically determined to obtain cell cycle distributions. Precision of DNA content measurement on LSC systems, expressed as a coefficient of variation (CV) of the mean DNA content of G1 CHO cells, is $2 \%$. DNA content information for any particular cell can be combined with the value of its most intense point of fluorecence, allowing additional discrimination between G0-noncycling and
G1-cycling cells, and between G2 interphase cells and mitotic cells. This assay is fully automated and can be performed on many individual samples. Apoptotic cells can be distinguished from live cells using the same principles.

Because the cell cycle assay requires only one of the cytometer's detectors, the others are free to measure related markers. For each pass of the laser beams, up to five different fluorescence and light scatter or light-loss measurements can be obtained. The additional information can include measurements such as the number of DNA strand breaks present in the nucleus, as determined by phosphorylated histone $\mathrm{H} 2 \mathrm{AX}$ antibodies. Activation states of molecules within cells can also be assayed with state-specific antibodies. For example, the commitment of a cell to apoptosis can be detected by monitoring caspase-3 cleavage. Additionally, cell morphology can be simultaneously monitored using labels for the cytoskeleton, such as actin or tubulin. This high-content approach is pertinent to apoptosis pathway research. The ability to survey specimens repeatedly over time further augments the utility of this technology (Fig. 2).

\section{Translocation studies}

Many cell cycle and signal transduction molecules originate in the cell cytoplasm and migrate to the nucleus; hence, translocation studies are important for drug discovery and development projects. LSC automatically quantifies and records images of subcellular constituent localization and translocation, so that drug effects can be monitored. Nuclear factor $\mathrm{\kappa B}$ and FISH probe spot evaluation represent some examples of this application.

\section{Live-cell studies}

Live-cell studies are greatly facilitated by LSC's inverted format, where cells can be analyzed in a wide range of carriers. LSC allows live-cell analysis with minimal sample disruption, and eliminates the need for specimen washing. This is particularly important in apoptosis applications, in which fragile, loosely adherent apoptotic cells may be lost during washing, leading to potentially ambiguous results. Live-cell analysis allows monitoring of functional effects, such as loss of mitochondrial membrane 
Figure 3 | Hepatotoxicological tissue analysis. Tissue sections from drug-treated animals were stained with antibodies to an induced protein, developed with the chromatic dye DAB and counterstained with hematoxylin. (a) Mosaic image of a scan area showing the localization of the protein. (b) Scattergram showing the blue versus red light-loss of random sampling units. Gates were drawn around regions defined in controls to discriminate between areas of no tissue, no DAB staining, and gradations of the DAB staining. (c) Cartesian map of the sampling elements from the same tissue section, showing the correlation of the quantitative elements with the protein distribution in the tissue. (d) Histograms of the random sampling elements showing the quantification of dose-dependent DAB staining. be analyzed simultaneously. a

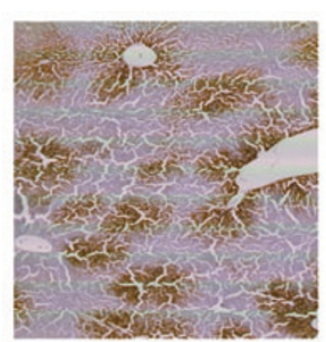

b

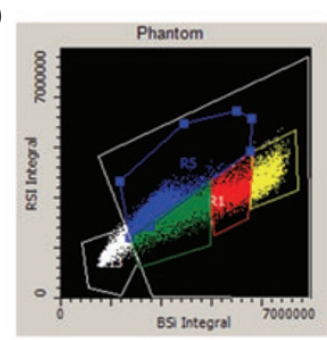

C

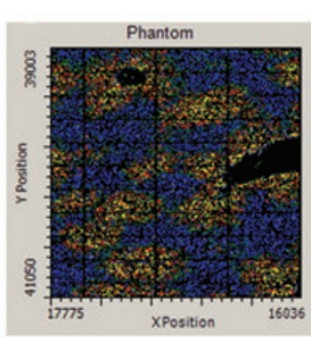

d

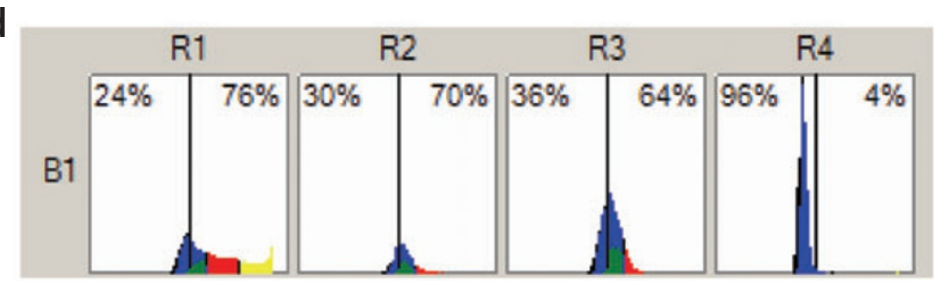

potential or abrogation of homotypic cell adhesion in colonyforming cells. A recent development allows researchers to first analyze live cells, and then remove them from the instrument to fix and restain with a new set of reagents for further analysis. The resultant image files can be merged, allowing the two data sets to

\section{FRET studies}

Cloning of the green fluorescent protein gene has led to a revolutionary new approach in cellular analysis, allowing the engineering of cells to produce their own fluorescent labels for defined targets of interest. The subsequent development of spectrally distinct variants, such as cyan and yellow fluorescent proteins, has led to the use of even more sophisticated techniques relying on fluorescence resonance energy transfer (FRET), a phenomenon that allows measurement of the proximity of molecules down to the angstrom level. This application has found its place in monitoring the formation of protein dimers, where each monomer is tagged with a fluorescent protein, and can also be applied to the study of single molecules. FRET data can be readily quantified in an automated fashion with relatively high throughput via LSC.

\section{Automated analysis of tissues and tissue microarrays (TMAs)}

LSC technology is well-suited for analysis of samples with preserved tissue architecture, such as tissue imprints, TMAs and tissue sections ${ }^{4}$. Automated tissue analysis is desirable to increase throughput and introduce objectivity. LSC allows simultaneous analysis of fluorescently and chromatically stained sections. Special segmentation methods based on random sampling are available to supplement nuclear segmentation. Unique image processing techniques correct for background fluorescence and spectral overlap of both fluorescent dyes and chromophores. These techniques make LSC technology particularly useful in pharmaceutical preclinical drug safety studies performed in animal models ${ }^{5}$, and in phase II and III clinical trials in which drug effectiveness and safety are tested on biopsy tissue samples ${ }^{6}$. Multiscale scanning is available to increase specimen throughput. First, a high-speed scout scan is performed to identify areas for rescans or to identify specific objects of interest within scan areas. This is followed by a high-resolution scan of selected areas to identify their contents and quantify constituents of interest. Resultant data can be displayed as mosaic images, scattergrams, histograms and expression maps (Fig. 3).

\section{Summary}

LSC is a unique technology that allows accurate cellular and tissue constituent quantification, combined with spatially based image analysis for more comprehensive specimen investigation. The development of LSC coincides with the genesis and broad availability of multicolored fluorescent tags and probes, and the increased commercial availability of highly specific antibodies. This mix promises to produce richly detailed data, quantifying highly intricate cellular patterns and functions. LSC can be used as a stand-alone platform, but also it complements other technologies such as FC and confocal microscopy. Some of the research areas that are now benefiting from the active use of LSC technology are systems biology; early-stage drug discovery-particularly target identification, lead optimization, predictive and investigative toxicology; biomarker discovery and validation, with application in phase II and phase III clinical trials; and immunophenotyping and immunohistochemistry analysis in clinical research.

1. Luther, E., Kamentsky, L., Henriksen, M. \& Holden, E. Next-generation laser scanning cytometry. Methods Cell Biol. 75, 185-218 (2004).

2. Huang, X., Okafuji, M., Traganos, F., Luther, E., Holden, E. \& Darzynkiewicz, Z. Assessment of histone H2AX phosphorylation induced by DNA topoisomerase I and II inhibitors topotecan and mitoxantrone and by the DNA cross-linking agent cisplatin. Cytometry A 58, 99-110 (2004).

3. Maruvada, P., Dmitrieva, N., East-Palmer, J \& Y Yen, P. Cell cycle-dependent expression of thyroid hormone receptor-beta is a mechanism for variable hormone sensitivity. Mol. Biol. Cell 15, 1895-1903 (2004).

4. Megyeri, A., Bacso, Z., Shields, A. \& Eliason, J. Development of a stereological method to measure levels of fluoropyrimidine metabolizing enzymes in tumor sections using laser scanning cytometry. Cytometry $A$ 64, 62-71 (2005).

5. Pruimboom-Brees, I. et al. Using laser scanning cytometry to measure PPAR-mediated peroxisome proliferation and oxidation. Tox. Path. 33, 86-91 (2005)

6. Davis, D. et al. Pharmacodynamic analysis of target inhibition and endothelial cell death in tumors treated with the vascular endothelial growth factor receptor antagonists SU5416 and SU6668. Clin. Cancer Res. 11, 678-689 (2005). 